J. Comp. Int. Sci. (2013) 4(3): xxxx-xxxx http://epacis.netjjis/PDF_JCIS/JCIS-0074.pdf jcis@epacis.net

@2013 PACIS (http://epacis.net)

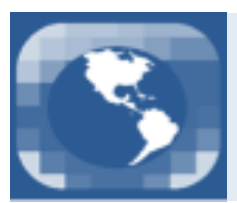

\title{
An exact solution for the capacitated multiple allocation hub location problem
}

\author{
Wesley G. de Almeida ${ }^{a 1}$, Edson L. F. Senne ${ }^{b}$ and Horacio H. Yanasse ${ }^{c}$ \\ ${ }^{a}$ Federal Institute of Minas Gerais (IFMG), São João Evangelista, MG, Brazil \\ ${ }^{b}$ São Paulo State University (UNESP), Guaratinguetá, SP, Brazil \\ ${ }^{c}$ Federal University of São Paulo (UNIFESP), São José dos Campos, SP, Brazil \\ Received on February 25, 2013 / Accepted on November 17, 2013
}

\begin{abstract}
The object of this work is to present an exact solution for the capacitated multiple allocation hub location problem. In order to accelerate the search for solutions, the Local Branching (LB) technique was employed. This technique is based on branch-and-cut methods and it also incorporates some ideas present in local search and metaheuristics.
\end{abstract}

Keywords: local branching, location, hubs, metaheuristics, computational mathematics.

\section{Introduction}

Transport companies are now facing the need to better plan their freight routes, because the mere definition of routes based on the source and target nodes can considerably increase the cost of transportation. The hub concept allows for a decrease in the total network cost.

A hub can be regarded as a central or terminal where cargo from different sources is aggregated to be later transported from the common hub to their final destination. Transport networks like this are called hub-and-spoke. Figure 1 shows two examples of huband-spoke networks. In the example on the left, each client can only be allocated to a single hub while in the example on the right a client can be allocated to more than one hub.

The Hub Location Problem (HLP) in a network consists in determining the number of consolidation installations, the localization of each one of them, and the allocation of the remaining network nodes (also called spokes or demand nodes) to the hubs so as to minimize the total cost of the operation, which may include the variable cost of transport (collec- tion, transfer, and distribution costs) and the fixed costs for facility location. These facilities may be factories, harbors, retail outlets, routine or emergency service points, post offices, waste incineration plants, medical centers, airports, communication antennas, schools, libraries among others (see [10], [3] and [5]).
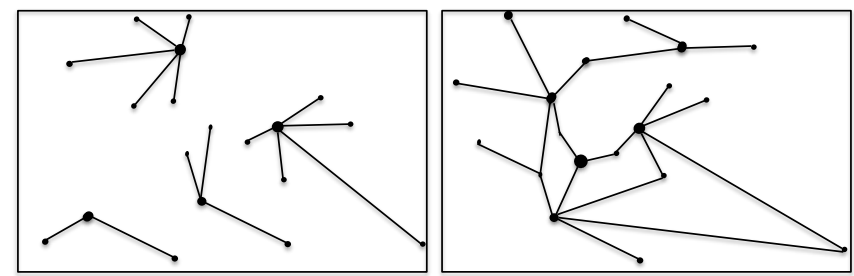

Figure 1: Example of networks with single (left) and multiple (right) allocation [6]

The HLP has several variations [1]. This paper aims at solving the capacitated multiple allocation HLP by using the Local Branching (LB) technique. This can be considered one of the most important problems due to its closeness to reality, and the use of the LB technique poses a great challenge for not having been used to solve hub location problems.

\footnotetext{
${ }^{1}$ E-mail Corresponding Author: wesley.almeida@ifmg.edu.br
} 


\section{Mathematical Model}

One of the first mathematical models for capacitated multiple allocation hub localization problem was proposed by Campbel [2]. However, in this study the F-CMAHLP mathematical model proposed by
Every et al. [4] was used, which corresponds to the model (1-6) below. This model was chosen because it uses less variables and it has proven effective when working with $n$ values greater than forty without exceeding memory capacity:

$$
\begin{array}{ll}
\min & \sum_{i} \sum_{k} \sum_{l} Y_{k l}^{i}\left(\lambda C_{i k}+\alpha C_{k l}\right)+\sum_{i} \sum_{k} \sum_{l} \delta C_{l j} x_{l j}^{i}+f_{k} H_{k} \\
\text { s.t. } & \sum_{l} x_{l j}^{i}=w_{i j}, \quad \forall i, j \in V, \\
& \sum_{i} \sum_{l} Y_{k l}^{i} \leq Q_{k} H_{k}, \quad \forall k \in V, \\
& \sum_{i} \sum_{k} Y_{k l}^{i} \leq \sum_{i} \sum_{j} w_{i j} H_{l}, \quad \forall l \in V, \\
& \sum_{k} Y_{k l}^{i}=\sum_{j} x_{l j}^{i}, \quad \forall i, l \in V, \\
& x_{l j}^{i}, Y_{k l}^{i} \geq 0, H_{k} \in\{0,1\} \quad \forall i, j, k, l \in V,
\end{array}
$$

The variables in this model are defined as follows:

- $C_{i j}$ is the distance between nodes $i$ and $j$;

- $Y_{k l}^{i}$ corresponds to the flow going through node $i$ via $k$ and $l$ hubs;

- whereas $x_{l j}^{i}$ determines the amount of flow originating at $i$ and ending at $j$ via the $l$ hub;

- $H_{k}$ is a decision variable that defines whether $k$ is a hub, $H_{k}=1$, or not, when $H_{k}=0$

- $V$ is the set of network nodes;

- $w_{i j}$ is the amount of flow transferred between $i$ and $j$ nodes;

- $\lambda, \alpha, \delta$ are, respectively, the collection, transfer, and distribution costs

- $f_{k}$ corresponds to the fixed cost for open a hub in $k$;

- And $Q_{k}$ corresponds to the maximum capacity of hub $k$.

For the mathematical model (1-6) the objective function (1) determines that the cost for hub location and allocation must be the lowest possible, taking into account the transportation costs and the fixed costs for the hub location. Constraints (2) establish that the sum of all installments from the flows originating in $i$ with destination in $j$ via hub $l$ must be equal to the total flow from $i$ to $j\left(w_{i j}\right)$. Constraints (3) ensure that the sum of the flows arriving at hub $k$ do not exceed its maximum capacity. For constraints (4) it is observed that the total sum of the flows arriving in $l$ must be less than or equal to the total flow associated with the selected hub $H_{l}$. Constraints (5) ensure that the total flow arriving at hub $l$ must be equal to the sum of all flows associated with hub $l$. Finally, constraints (6) define the integrality constraints of the decision variables.

\section{Local Branching Strategy}

The Local Branching (LB) technique proposed by Fischetti and Lodi [7] employs a commercial solver to effectively explore (at a tactical level) suitable solution subspaces (at a strategic level) in a local branch structure.

The procedure has the spirit of local search metaheuristics, but the neighborhoods are achieved by inserting local branching cuts in the integer programming model that describes the problem. This solution strategy is inherently exact and it switches between strategic ramifications to set solution neighborhoods and tactical ramifications to explore these neighborhoods. The outcome is a completely general scheme that anticipates improvements in incumbent solutions, hence generating high-quality solutions in the initial stages of the enumeration tree, thus reducing the computational time.

Consider the mathematical model (7-11) for com- 
binatorial optimization problems with binary variables:

$$
\begin{aligned}
F(x)=\min C^{T} x & \\
\text { s.t. } & A x \geq b, \\
& X_{i} \in B, \\
& X_{i} \in Z_{+}, \\
& X_{i} \in R_{+}, \\
& A \in Q^{M \times N}, b \in Q^{M}, c \in Q^{N} .
\end{aligned}
$$

The set of variables indices $\mathrm{I}:=1,2,3, \ldots, \mathrm{n}$ is partitioned in the sets $(\mathrm{B}, \mathrm{Z}, \mathrm{R})$, where $B \in \emptyset$ is the set of indices of the binary variables and $\mathrm{Z}$ and $\mathrm{R}$ are the sets of indexes of integer and continuous variables, respectively.

The LB algorithm works with the concept of a feasible solution neighborhood, defined as follows: given a feasible solution $\bar{x}$ for (7-12), $S=\left\{i \in B: \bar{x}_{i}=1\right\}$ is defined as the binary support for $\bar{x}[7]$. Given a parameter $k$ specifies to the K-OPT neighborhood of $\bar{x}$ is defined, denoted by $N(\bar{x}, k)$ as the set of feasible solutions that besides satisfying (8)-(12) also satisfy the Local Branching constraint:

$$
\Delta(x, \bar{x})=\sum_{j \in S}\left(1-x_{j}\right)+\sum_{j \in B \backslash S} x_{j} \leq k
$$

The Local Branching constraint can be used as a branching criterion in a Branch-and-Bound (B\&B) enumeration scheme. Given a feasible solution $\mathrm{x}$, the solution space associated with the current node can be partitioned into (i) $\Delta(x, \bar{x}) \leqslant k$ (left branch) and (ii) $\Delta(x, \bar{x}) \geqslant k+1$ (right branch). The parameter $\mathrm{k}$ must be chosen so that the search space of the subproblem associated with the left bundle-branch is large enough to contain the best feasible solution $\mathrm{x}$ and at the same time small enough so that it is easier to be solved than the problem associated with its father.

This constraint therefore requires that $k$ is the longest distance between viable neighbors. This Local branching constraint may be used in an enumerative method as a cramification criterion. Figure 2 shows the basic idea of the LB technique. The method starts the search from an initial reference solution and to each level of the tree a new Local Branching constraint is added so that a solver can explore the solution neighborhoods present in each branch. The tree adds local constraints as improvement occurs in the current solutions.

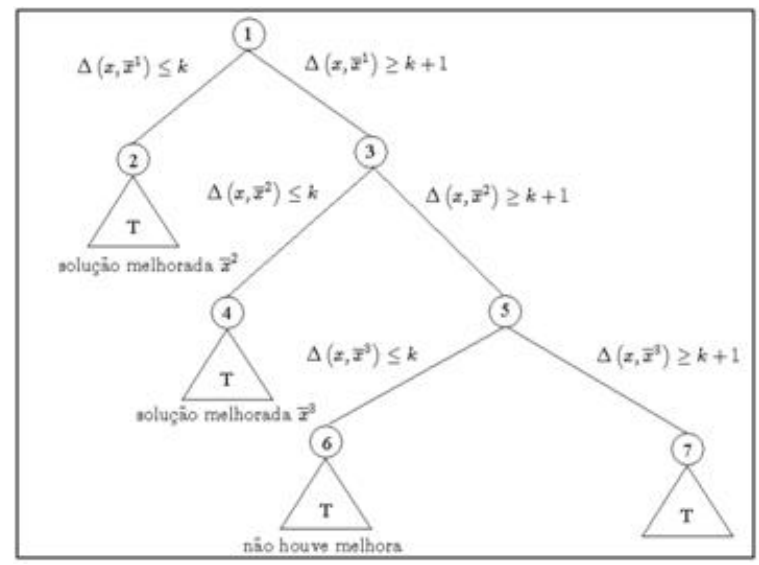

Figure 2: Enumeration tree LB.

\section{Proposed Methodology}

The LB method presented depends on a good initial reference solution. In this work a Local Search strategy capable of solving the capacitated hub location problem so as to generate an initial solution was developed. The local search method proposed, based on the Hill Climbing method, is a local search technique that explores the solution space by means of systematically switching between different neighborhood structures. The methodology consists in the following: a randomly generated solution is subject to a small disturbance, and if this movement generates an improvement in the current solution, it is accepted. The pseudocode is presented in Figure 3. A possible solution used by the Local Search (LS) method corresponds to a vector formed by $n^{2}$ positions, each corresponding to a pair (source, destination), which in turn is associated with a route (source-hub, destination hub).

\section{Procedure Local Search}

Let $s^{0}$ be initial solution and $r$ the number of neighborhood structures

$s \leftarrow s^{0} \quad$ Current solution\}

$k \leftarrow 1 ;$ \{Type of neighborhood structure\}

while (Stopping criterion not satisfied) do

Generate a neighboring any s' $\in N(s)$

if $\left(f\left(s^{\prime}\right)<f(s)\right)$

then $s \leftarrow s^{\prime}$;

$k \leftarrow k+1$

End-if

End-while

Returne $s$

End-procedure

Figure 3: Hill climbing pseudocode. 
Figure 4 shows a possible solution for the problem, in which the number of network nodes is $n=5$ and the number of hubs is $p=3$. The positions of vector $\mathrm{V}$ range from 0 to 24 to represent the possible combinations of source-destination pairs, and each position can store a value from 0 to 8 to represent all possible routes going from one hub to the other. When analyzing the position 3 for vector $\mathrm{V}$, for instance, it is possible to notice that a freight leaving the source-destination pair $[3]=(1,4)$ must be sent via hubs $[7]=(3,2)$ by the following relationship: source-destination $[i]=((i \% n)+1,(i \operatorname{div} n)+1)$ and hubs $[i]=((v[i] \% p)+1,(v[i] \operatorname{div} p)+1)$, where $\%$ represents the remainder and the div the integer division. Note that in the table of possible hubs $k$ and $l$ represent the vector index of the chosen hubs. This vector stores which points from 1 to $\mathrm{n}$ were chosen to be hubs.

The implemented LB method initially generates the hubs randomly and then associates each vector $\mathrm{V}$ position to the route that goes via the nearest pair of hubs according to the pseudocode presented in Figure 5. To calculate the cost $C_{i j k m}$ simply add the collection, transfer, and distribution costs multiplied by the corresponding distance $\left(C_{i j k m}=\lambda d_{i k}+\alpha d_{k m}+\delta d_{m j}\right)$.

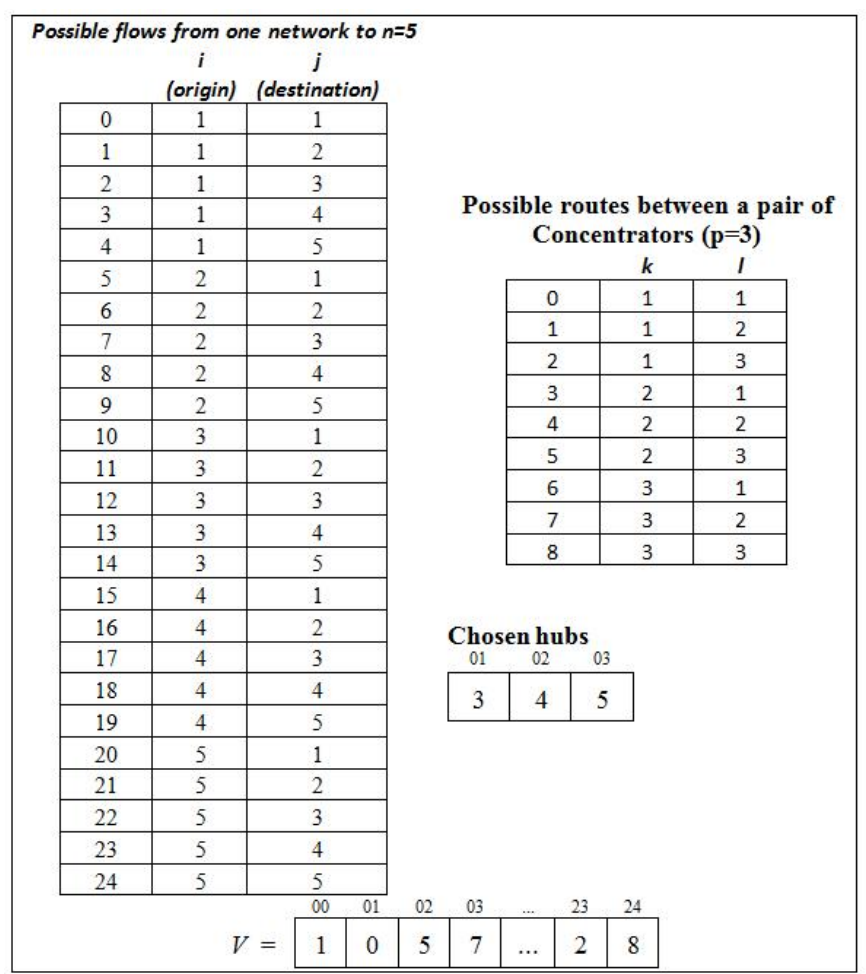

Figure 4: Representation of a solution $\mathrm{N}=5$ e $\mathrm{P}=3$.

After generating vector $V$ with its allocation routes from the nearest hubs, it goes through an evaluation function responsible for scoring the found so- lution. This function yields the solution values according to the object function. The only exception happens when a generated solution exceeds the hub flow capacity constraint, in which case the evaluation function assigns a penalty so that this solution is discarded in the method search process.

In order to develop the local search method four neighborhoods were defined:

- Realocate Hub: chooses a non-hub node $A$ and a hub node $B$ to make a switch, so that $A$ becomes a hub and $B$ a non-hub node;

- Add Hub: this procedure adds a non-hub element to the set of solution hubs;

- Delete Hub: chooses a hub from the set of solution hubs to turn it into a non-hub element;

- Swap Route: randomly chooses two routes from vector $\mathrm{V}$ and swaps them.

After moves 1, 2, or 3, all elements from vector $\mathrm{V}$ are reallocated to the nearest hubs route; The developed LB method has the results from the local search heuristic as an initial reference solution and the LB local ramifications use the binary variable $\mathrm{H}$ from model (1-6) proposed by Every et al. [4].

\section{Results}

The verification of the efficacy of the implemented method was based on the AP data set [5]. The AP (Australian Post) data set was proposed by Ernst and Krishinamoorthy [5]. The values for the collection, transfer, and distribution costs are 3, 0.75 and 2 respectively. The tests were run on a Core 2-Duo PC with 2 GB of RAM on a Linux operating system with CPLEX 12. The $k$ value of the local branching constraint was set at 30 .

In this work, the time spent by the CPLEX and the LB to find the optimal solutions was analyzed. Table 1 contains the following columns:

- Test: describes the test instance used;

- Sol: indicates the solution found by the implemented methods;

- TE: corresponds to the runtime of the algorithm (in seconds) until the stop criterion is reached;

- Optimal: indicates the optimal solution found in the literature;

- VAR: corresponds to the difference between the CPLEX runtime and the LB runtime; 
- GapIni: displays the initial gap of the search for solution;

- LS1: local search method one, used to generate the initial solution for the CPLEX;

- LS2: local search method two, the reference solution for LB.

Table 1: Results CPLEX x LB.

\begin{tabular}{c|c|cr|rr|rr|rr|c}
\hline \multirow{2}{*}{ Test } & \multirow{2}{*}{ Optimal } & \multicolumn{2}{|c|}{ LS $_{1}$} & \multicolumn{2}{|c|}{ CPLEX } & \multicolumn{2}{c|}{ LS $_{2}$} & \multicolumn{2}{|c|}{ LB } & \multirow{2}{*}{ VAR } \\
\cline { 3 - 9 } & & SOL & TE & GapIni & TE & SOL & TE & GapIni & TE & \\
\hline AP10LL & 221032.73 & 221032.73 & 2.24 & 66.80 & 2.63 & 221032.73 & 0.25 & 66.80 & 2.53 & 0.1 \\
AP10LT & 246495.04 & 246495.04 & 2.27 & 45.39 & 1.16 & 246495.04 & 0.3 & 45.39 & 1.26 & -0.1 \\
AP10TL & 257558.08 & 257558.08 & 2.22 & 62.87 & 3.22 & 257558.08 & 0.29 & 62.87 & 2.86 & 0.36 \\
AP10TT & 257558.08 & 257558.08 & 2.22 & 41.76 & 1.44 & 257558.08 & 0.32 & 41.76 & 1.48 & -0.04 \\
\hline AP20LL & 230385.45 & 230385.45 & 10.73 & 60.95 & 197.73 & 230385.45 & 1.12 & 60.95 & 122.58 & 75.15 \\
AP20LT & 246433.75 & 259118.36 & 10.71 & 45.43 & 82.1 & 263789.97 & 1.12 & 46.39 & 83.58 & -1.48 \\
AP20TL & 266877.48 & 266877.48 & 10.71 & 54.09 & 165 & 266877.48 & 1.13 & 54.09 & 121.97 & 43.03 \\
AP20TT & 287574.76 & 311234.66 & 11.82 & 37.30 & 88.75 & 320391.35 & 1.17 & 39.09 & 63.63 & 25.12 \\
\hline AP25LL & 233636.70 & 239324.62 & 19.67 & 60.20 & 927.2 & 240191.40 & 2.06 & 60.20 & 923.09 & 4.11 \\
AP25LT & 263760.55 & 274103.67 & 19.83 & 36.78 & 598.55 & 277389.54 & 2.02 & 36.78 & 526.16 & 72.39 \\
AP25TL & 305982.08 & 305982.09 & 19.85 & 50.84 & 633.31 & 305982.09 & 1.99 & 50.84 & 763.32 & -130.01 \\
AP25TT & 333221.74 & 347745.52 & 20.04 & 26.81 & 313.57 & 347745.52 & 2.03 & 26.81 & 405.8 & -92.23 \\
\hline AP40LL & 238313.46 & 238313.46 & 79.74 & 55.02 & 67365.82 & 238313.46 & 8.02 & 55.02 & 17129.37 & 50236.45 \\
AP40LT & 261547.13 & 289209.91 & 80.29 & 40.78 & 7317.4 & 289209.91 & 7.99 & 40.78 & 5060.82 & 2256.58 \\
AP40TL & 297204.12 & 300779.35 & 80.35 & 41.16 & 5153.49 & 300779.35 & 8.02 & 41.16 & 4509.52 & 643.97 \\
AP40TT & 339965.86 & 428933.72 & 79.43 & 34.65 & 1615.62 & 405607.94 & 7.97 & 30.89 & 1765.6 & -149.98 \\
\hline AP50LL & 234399.92 & 234399.92 & 144.42 & 56.09 & 296983.9 & 234399.92 & 14.67 & 56.09 & 74485.32 & 222498.6 \\
AP50LT & 264999.71 & 293361.33 & 145.47 & 41.95 & 68194.35 & 316580.88 & 14.49 & 46.21 & 22317.31 & 45877.04 \\
AP50TL & 314845.55 & 318334.12 & 144.76 & 40.91 & 20286.58 & 318334.12 & 14.9 & 40.91 & 17167 & 3119.58 \\
AP50TT & 391287.29 & 485702.15 & 143.96 & 34.43 & 8022.92 & 501709.43 & 14.67 & 36.52 & 9742.83 & -1719.91 \\
\hline
\end{tabular}

The difference between LS1 and LS2 is the stopping criterion. 100,000 iterations were made for LS1 while 1,000 were made for LS2.

In order to obtain the results presented in Table 1, both the CPLEX and the LB used the descent method to generate an initial solution. From the test results it is possible to identify that there was a reduction of the runtime in $80 \%$ of the cases.

\section{Conclusions}

The capacitated hub location problem is a relevant Combinatorial Optimization problem as it occurs in several practical situations where transport of some entity (people, data, goods, etc.) must undergo an aggregation process before being distributed to its destination. Good solutions for this problem may pose significant economic gains for several business sectors.

The HLP is known to be NP-hard class [8] and exact algorithms to determine the optimal solution to this problem are a major challenge since they require methods that demand great computational effort, which may be impractical for large scale problems.

Nevertheless, studies and tests conducted with the Local Branching (LB) technique have shown consid- erable ability (have yielded good results) in the reduction of search time for optimal solutions. It was also observed that, in smaller instances, during Local search heuristic it generated near-optimal solutions.

During the test runs it was observed that the main difficulty identified by the proposed method was to reduce the time to prove that the found solution was optimal.

Acknowledgments:The authors would like to thank CAPES for financial support and the anonymous reviewers for their suggestions.

\section{References}

[1] Alumur, S. and Kara, B. Y.: Network hub location problems: The state of the art. European Journal of Operational Research 190, 1-21 (2008)

[2] Campbell, J.E.: Integer programming formulations of discrete hub location problems. European Journal of Operational Research 72, 387405 (1994).

[3] Cunha, C.B.; Silva, M.R.: A genetic algorithm for the problem of configuring a hub-and-spoke 
network for a LTL trucking company in Brazil. European Journal of Operational Research 179, 747-758 (2007).

[4] Ebery, J; Krishnamoorthy, M; Ernst, A; Boland, N.: The Capacitated multiple allocation hub location problem: Formulations and algorithms. European Journal of Operational Research 120, 614-631 (2000).

[5] Ernst, A.T. and Krishinamoorthy, M.: Efficient algorithms for the uncapacited single allocation p-hub median problem. Location Science 4(3), 139-154 (1996).

[6] Ernst, A.T and Krishnamoorthy, M.: Exact and heuristic algorithms for the uncapacitated multiple allocation p-hub median problem. European Journal of Operational Research 104(1), 100-112 (1998).
[7] Fischetti, M. and Lodi, A.: Local branching. Mathematical Programming 98 (1-3), 23-47 (2003).

[8] Garey, M. R. and Johnson, D. S.: Computers and Intractability: A Guide to the Theory of NP-Completeness. San Francisco: W.H. Freeman (1979).

[9] Mladenovic, N. and Hansen, P.: Variable neighborhood search. Comps. \& Opns 24(11), 10971100 (1997).

[10] O'Kelly, M.: A quadratic integer program for the location of interacting hub facilities. European Journal of Operational Research 32, 393404 (1987). 